\title{
Natural Convection Induced by Diurnal Heating and Cooling in a Reservoir with Slowly Varying Topography*
}

\author{
Chengwang LEI** and John C. PATTERSON**
}

\begin{abstract}
This study is concerned with natural convection in a reservoir with slowly varying topography in response to diurnal heating and cooling due to heat transfer through the water surface. In the daytime phase, heat is transferred into the water body through absorption of solar radiation; and in the night-time phase, heat is transferred out of the water body through heat loss from the water surface. An unsteady model is formed and solved numerically in order to investigate the transient flow response in the reservoir. Two different scenarios with shallow and deep waters respectively, based on the comparison between the maximum water depth and the penetration depth of the solar radiation, are considered. The numerical results reveal that there is a distinct time lag in the response of the overall flow to the switches of the thermal forcing, and the lag time depends on the Grashof number. It is also found that thermal instabilities play an important role in breaking the residual circulation and reversing the flow in deep waters.
\end{abstract}

Key Words: Natural Convection, Reservoir, Exchange Flow, Periodic Forcing, Topography

\section{Introduction}

Large-scale convective circulations in response to diurnal heating and cooling due to heat transfer through the water surface take place in the sidearm of reservoirs or shallow littoral waters with gently sloped bottoms. These convective circulations play an important role in the transport of nutrients and pollutants between the shallow region and the bulk deep water ${ }^{(1)-(3)}$. In a typical diurnal cycle, the water body is subject to solar radiation during the daytime and heat loss through the water surface during the night-time. When the shallow and deep regions are exposed to a uniform rate of surface cooling under the night-time condition, the shallow water cools faster than the deep water. The difference in the cooling rate then results in a horizontal temperature gradient which in turn drives a downward dense gravity current along the sloping bottom $^{(1),(4)-(6)}$. A similar but converse statement can be made for the daytime heating condition ${ }^{(7)-(9)}$. Put simply for the day-time case, the vertically averaged volumetric rate of absorption of a spatially uniform incident radiation flux is greater in shallow parts than that in deeper parts, re-

\footnotetext{
Received 13th February, 2006 (No. 06-5021)

** School of Engineering, James Cook University, Townsville, Queensland 4811, Australia.

E-mail: Chengwang.Lei@jcu.edu.au;

John.Patterson@jcu.edu.au
}

sulting in relatively warm shallow regions compared with deeper regions. A convectively driven circulation results, with a warm upward flow along the sloping bottom and a horizontal outflow along the water surface.

In general the flow response in reservoirs is characterized by unsteady thermal forcing and large scale circulations in field situations. Of particular interest is the response of the convective circulation to the switches between the daytime heating by solar radiation and nighttime cooling due to heat loss through the water surface. Field observations ${ }^{(10),(11)}$ have shown that the circulation in a reservoir sidearm or the littoral region of a lake is not in phase with the thermal forcing, and thus the flow is against the prevailing pressure gradient. Farrow \& Patterson $(1993)^{(2)}$ confirmed the lag of the flow response to the switches of thermal forcing based on a zero-order asymptotic solution to a simplified diurnal model, and reported that the time lag can be up to 12 hours. Later, Farrow $(2004)^{(3)}$ extended the asymptotic solution of Farrow \& Patterson $(1993)^{(2)}$ to a higher order and included arbitrary bathymetry.

In the model adopted in Farrow \& Patterson (1993) ${ }^{(2)}$ and Farrow $(2004)^{(3)}$, a heat flux representing both daytime heating (positive) and night-time cooling (negative) is uniformly distributed over the entire local water depth. This model may be appropriate for the night-time cooling phase when strong vertical mixing occurs due to con- 
vective instabilities. However, it is not an accurate representation of the daytime heating process by solar radiation. A distinctive feature of the daytime heating by solar radiation is that the radiative energy is absorbed, and thus attenuated by the water body, according to a decaying exponential function of the water depth ${ }^{(7)-(9),(12),(13)}$. Therefore, the penetration depth of the solar radiation in water is limited, and is quantified by the inverse of the attenuation coefficient of the solar radiation relevant to the wavelength of the radiation and the turbidity of the water. Accordingly, water bodies may be classified into shallow waters and deep waters, depending on the comparison between the maximum water depth and the penetration depth of the solar radiation. In shallow waters, the maximum water depth is less than the penetration depth of the solar radiation, and thus a significant amount of radiation entering from the water surface will reach the bottom. This residual radiation reaching the bottom is either scattered, or reflected, or absorbed by the bottom. Previous investigations by Lei \& Patterson ${ }^{(7)-(9),(12),(13)}$ have revealed that the residual radiation at the bottom is responsible for driving a convective circulation in shallow waters.

In the present study, the transient flow response to periodic heating and cooling in a reservoir with slowly varying topography is investigated with an improved diurnal model, which takes into account the different natures of thermal forcing in the daytime and at the night-time. Two different scenarios with the maximum water depth less and greater than the penetration depth of the solar radiation, respectively, are considered. The complete set of governing equations is solved numerically, which enables an examination of the effects of flow instabilities on the transient flow response.

The rest of the document is organized as follows. Section 2 introduces the details of the diurnal model and numerical procedures. Section 3 discusses qualitatively the flow response to the diurnal forcing in both shallow and deep waters, and section 4 presents quantitative data with regard to the diurnal flow response. Finally, section 5 summarizes major findings of the present investigations.

\section{Diurnal Model and Numerical Procedures}

\subsection{Computational domain and thermal forcing}

A two-dimensional (2D) reservoir model consisting of a region with a sloping bottom and an adjacent region of a uniform water depth is considered here (see Fig. 1). Here, $h$ is the maximum water depth in the reservoir, and $x$ and $y$ are the horizontal and vertical coordinates with origin at the tip of the domain. Previous investigations have suggested that $2 \mathrm{D}$ numerical models can reproduce major flow features observed in both heating and cooling experiments $^{(4),(5),(7),(12)}$.

Two types of thermal forcing are constructed for the reservoir model in order to simulate the diurnal cycle in

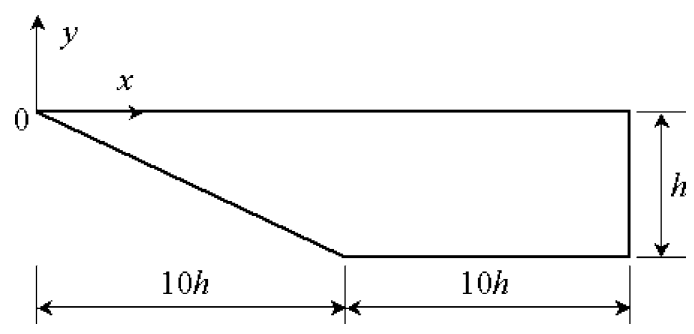

Fig. 1 Sketch of the flow domain and coordinate system

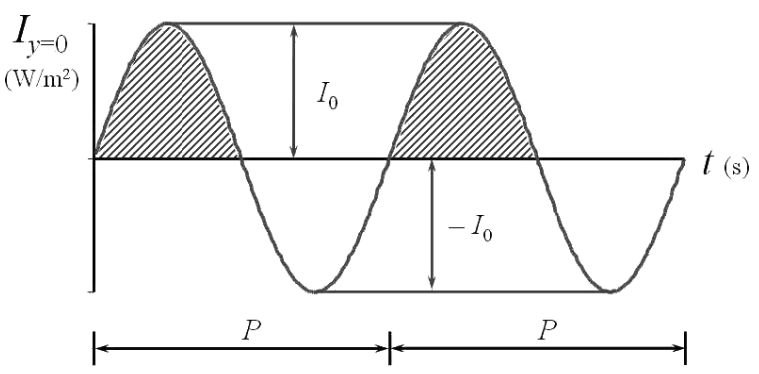

Fig. 2 Illustration of the periodic thermal forcing

field situations. They are both represented by the following sinusoidal function

$$
I_{y=0}=I_{0} \sin (2 \pi t / P),
$$

and are plotted in Fig. 2. Here, $I_{y=0}$ is the time-dependent intensity of the thermal forcing at the water surface, $I_{0}$ the peak intensity, $P$ the period of the thermal forcing, and $t$ is the time. Equation (1) implies that the thermal forcing is uniform across the water surface.

During the first half of every forcing cycle (the shaded parts in Fig. 2), $I_{y=0}$ is positive, representing the solar radiation entering through the water surface during the daytime. The solar radiation with a time-varying intensity of $I_{0} \sin (2 \pi t / P)$ is then absorbed by the water body according to the Beer's law ${ }^{(7),(8),(12) \text { : }}$

$$
I=I_{y=0} \cdot e^{y \eta} \quad(y \leq 0),
$$

where $I$ is the radiation intensity at a given water depth $(-y)$, and $\eta$ is the attenuation coefficient of the solar radiation in water. The attenuation coefficient is dependent on the wavelength of the solar radiation and the turbidity of the water. In the present study, we are concerned only with the spectrally integrated effect of the solar radiation, and thus the attenuation coefficient of the solar radiation is represented by a single spectrally averaged value which can be regarded as a constant ${ }^{(7)-(9),(12),(13)}$. The typical value of the attenuation coefficient relevant to field situations is of the order of $O\left(1 \mathrm{~m}^{-1}\right)$. It is also assumed in this study that any radiation reaching the bottom is fully absorbed by the bottom and the absorbed energy is then fully released back into the water as a boundary heat flux. Moreover, it is assumed that the heat loss through the water surface during the heating phase is negligible, and all the radiation entering through the water surface is ultimately absorbed by the water. 
During the second half of the forcing cycle (the unshaded parts in Fig. 2), $I_{y=0}$ is negative, representing the heat loss through the water surface during the night-time. In this case, all the rigid boundaries are assumed adiabatic. Since there is no other form of energy entering or leaving the water body either in the daytime or at the night-time except that through the water surface, the present diurnal model ensures that the total heat absorbed by the water body during the daytime heating phase is in a balance with the total heat released by the water body during the nighttime cooling phase. Moreover, the representation of the two different types of thermal forcing by a common sinusoidal function (Eq. (1)) ensures a smooth transition from one type of thermal forcing to the other. The combination of these two types of thermal forcing allows us to simulate an entire diurnal cycle.

\subsection{Governing equations and boundary conditions}

The flow and temperature evolutions in the reservoir are governed by the two-dimensional Navier-Stokes and energy equations given below, in which the Boussinesq assumptions have been made:

$$
\begin{aligned}
& \frac{\partial u}{\partial x}+\frac{\partial v}{\partial y}=0 \\
& \frac{\partial u}{\partial t}+u \frac{\partial u}{\partial x}+v \frac{\partial u}{\partial y}=-\frac{1}{\rho_{0}} \frac{\partial p}{\partial x}+v \nabla^{2} u \\
& \frac{\partial v}{\partial t}+u \frac{\partial v}{\partial x}+v \frac{\partial v}{\partial y}=-\frac{1}{\rho_{0}} \frac{\partial p}{\partial y}+v \nabla^{2} v+g \beta\left(T-T_{0}\right) \\
& \frac{\partial T}{\partial t}+u \frac{\partial T}{\partial x}+v \frac{\partial T}{\partial y}=\alpha \nabla^{2} T+S r
\end{aligned}
$$

where $u$ and $v$ are the velocity components along $x$ and $y$ direction respectively, $p$ the pressure, $T$ the temperature, $\rho_{0}$ the density at the reference temperature $T_{0}, g$ the acceleration due to gravity, $v$ the kinematic viscosity, $\alpha$ the thermal diffusivity, and $\beta$ the thermal expansion coefficient. $\mathrm{Sr}$ is the internal heat source term quantifying the volumetric absorption of the solar radiation by the water body under the daytime condition, and is set to zero for the night-time cooling phase. Thus,

$$
S r=\left\{\begin{array}{cc}
\frac{I_{y=0}}{\rho_{0} C_{p}} \eta e^{y \eta} & \text { if } \sin (2 \pi t / P) \geq 0 \\
0 & \text { if } \sin (2 \pi t / P)<0
\end{array}\right.
$$

where $C_{p}$ is the specific heat of water.

The governing equations are subject to the following initial and boundary conditions:

(1) Initially $(t=0)$, the water is motionless and isothermal. That is

$$
\begin{aligned}
& u=v=0 \\
& T=T_{0}
\end{aligned}
$$

(2) At the deep end $(x=20 h)$, an adiabatic and rigid non-slip boundary condition applies, i.e.

$$
\begin{aligned}
& u=v=0 \\
& \frac{\partial T}{\partial x}=0
\end{aligned}
$$

The rigid non slip, adiabatic boundary conditions for the deep end are chosen for simplicity. Extensive numerical tests have shown that only the region in the immediate vicinity of the boundary is influenced by the presence of the boundary, and for the purposes of this paper the assumption of a rigid boundary is justified.

(3) The water surface $(y=0)$ is assumed to be stressfree, and thus the velocity boundary condition is written as

$$
v=0, \frac{\partial u}{\partial y}=0 .
$$

The thermal boundary condition at the water surface varies from daytime to night-time, and is given by

$$
-k \frac{\partial T}{\partial y}=\left\{\begin{array}{cc}
0 & \text { if } \sin (2 \pi t / P) \geq 0 \\
-I_{0} \sin (2 \pi t / P) & \text { if } \sin (2 \pi t / P)<0
\end{array}\right.
$$

where $k$ is the thermal conductivity of water at the reference temperature.

( 4 ) The sloping boundary and horizontal bottom are rigid and non-slip (i.e. $u=v=0$ ). The thermal boundary condition on the bottom also varies from daytime to nighttime, and is given by

$$
-k \frac{\partial T}{\partial n}=\left\{\begin{array}{cl}
I_{y=0} e^{y \eta} & \text { if } \sin (2 \pi t / P) \geq 0 \\
0 & \text { if } \sin (2 \pi t / P)<0
\end{array}\right.
$$

where $n$ is the direction normal to the bottom.

\subsection{Normalization}

The governing equations together with the initial and boundary conditions are normalized using the following scales:
Length $(x, y)$ :
Velocity $(u, v)$ :
$\sim h$
Time $(t, P)$ :
$\sim \alpha / h$
Temperature variation $\left(T-T_{0}\right)$ :
$\sim h^{2} / \alpha$
Pressure gradient $(\partial p / \partial x, \partial p / \partial y)$ :
$\sim I_{0} h / k$
Attenuation coefficient $(\eta)$ :
$\sim \rho_{0} g \beta I_{0} h / k$
$\sim 1 / h$.

The normalized governing equations are rewritten as:

$$
\begin{aligned}
& \frac{\partial u}{\partial x}+\frac{\partial v}{\partial y}=0 \\
& \frac{\partial u}{\partial t}+u \frac{\partial u}{\partial x}+v \\
& \frac{\partial v}{\partial t}+u \frac{\partial v}{\partial x}+v \\
& \frac{\partial T}{\partial t}+u \frac{\partial T}{\partial x}+ \\
& \text { Sr }=\left\{\eta e^{y \eta}\right. \text { s } \\
& \text { and } \operatorname{Pr} \text { are the } \\
& G r=\frac{g \beta I_{0} h^{4}}{v^{2} k} \\
& \operatorname{Pr}=\frac{v}{\alpha}
\end{aligned}
$$$$
\frac{\partial u}{\partial t}+u \frac{\partial u}{\partial x}+v \frac{\partial u}{\partial y}=-\left(\operatorname{Pr}^{2} G r\right) \frac{\partial p}{\partial x}+\operatorname{Pr} \nabla^{2} u
$$$$
\frac{\partial v}{\partial t}+u \frac{\partial v}{\partial x}+v \frac{\partial v}{\partial y}=-\left(\operatorname{Pr}^{2} G r\right) \frac{\partial p}{\partial y}+\operatorname{Pr} \nabla^{2} v+\left(\operatorname{Pr}^{2} G r\right) T
$$$$
\frac{\partial T}{\partial t}+u \frac{\partial T}{\partial x}+v \frac{\partial T}{\partial y}=\nabla^{2} T+S r
$$

where the internal heat source term $\mathrm{Sr}$ is now rewritten as:

$$
S r=\left\{\begin{array}{cc}
\eta e^{y \eta} \sin (2 \pi t / P) & \text { if } \sin (2 \pi t / P) \geq 0 \\
0 & \text { if } \sin (2 \pi t / P)<0
\end{array}\right.
$$

$G r$ and $P r$ are the Grashof and Prandtl numbers defined as: 
All the variables in Eqs. (15) - (19) are now dimensionless. The initial and boundary conditions are also normalized accordingly. The normalized initial and boundary conditions are summarized below:

( 1 ) Initially $(t=0)$,

$$
\begin{aligned}
& u=v=0 . \\
& T=0 .
\end{aligned}
$$

(2) At the end wall $(x=20 h)$,

$$
\begin{aligned}
& u=v=0 \\
& \frac{\partial T}{\partial x}=0
\end{aligned}
$$

(3) At the water surface $(y=0)$,

$$
\begin{aligned}
& v=0, \frac{\partial u}{\partial y}=0 \\
& -\frac{\partial T}{\partial y}=\left\{\begin{array}{cc}
0 & \text { if } \sin (2 \pi t / P) \geq 0 \\
-\sin (2 \pi t / P) & \text { if } \sin (2 \pi t / P)<0
\end{array}\right.
\end{aligned}
$$

(4) At the sloping and horizontal bottom,

$$
\begin{aligned}
& u=v=0 \\
& -\frac{\partial T}{\partial n}=\left\{\begin{array}{cl}
e^{y \eta} \sin (2 \pi t / P) & \text { if } \sin (2 \pi t / P) \geq 0 \\
0 & \text { if } \sin (2 \pi t / P)<0
\end{array}\right.
\end{aligned}
$$

All the variables in Eqs. (22) - (29) are also dimensionless.

\subsection{Numerical scheme}

The normalized governing equations together with the initial and boundary conditions are solved using a finite difference scheme. Standard second-order central differencing is used for all spatial derivatives except the non-linear terms in the momentum and energy equations, which are approximated with a modified second-order upwind scheme. The time integration for the velocity components and temperature is by a second-order time accurate backward differencing scheme. Further details of the numerical schemes can be found in Lei \& Patterson ${ }^{(12)}$.

\subsection{Numerical parameters}

Since the present investigation is relevant to reservoirs or lakes with water as the medium, the Prandtl number is fixed at 7 . The numerical simulations are based on a laboratory scaled model with a full length of $2 \mathrm{~m}$, a maximum water depth of $0.1 \mathrm{~m}$ and a bottom slope of 0.1 in the sloping region. An achievable experimental condition has a peak intensity of approximately $50 \mathrm{~W} / \mathrm{m}^{2}$ and a cycle period of approximately 4 hours for the thermal forcing. Accordingly, the Grashof number is calculated to be $1.73 \times 10^{7}$ based on a room temperature of $20^{\circ} \mathrm{C}$, and the dimensionless period of the thermal forcing is set to 0.2.

Two sets of simulations are conducted in this study. In the first set of simulations, we consider shallow waters with a maximum water depth less than the penetration depth of the solar radiation (i.e. $h<\eta^{-1}$ ). In this case, the dimensionless attenuation coefficient is set to 0.62 (i.e. $h \eta=0.62$ in dimensional form) according to the experimental measurement reported in Lei \& Patterson ${ }^{(12)}$. A less-than-unity value for the dimensionless attenuation coefficient implies that a significant portion of the radiation entering through the water surface will penetrate all the way through the water depth and reach the bottom (refer to Eq. (2)). For the purpose of examining the dependence of the flow response on the Grashof number, two Grashof number cases with $G r=10^{6}$ and $1.73 \times 10^{7}$ are calculated.

In the second set of simulations, we consider deep waters with a maximum water depth greater than the penetration depth of the solar radiation (i.e. $h>\eta^{-1}$ ). The dimensionless attenuation coefficient of the solar radiation is set to 5 (i.e. $h \eta=5$ in dimensional form), indicating that the maximum water depth is about 5 times the penetration depth of the solar radiation. Simulations are carried out for three different Grashof numbers of $G r=10^{4}, 10^{5}$ and $10^{6}$, respectively.

The table below summarizes all the numerical parameters for the simulations.

\begin{tabular}{|c|c|c|}
\hline $\begin{array}{c}\text { Normalized attenuation coefficient } \\
(\eta)\end{array}$ & $\begin{array}{c}\text { Period of thermal forcing } \\
(P)\end{array}$ & $\begin{array}{c}\text { Grashof numbers } \\
(\mathrm{Gr})\end{array}$ \\
\hline 0.62 (Shallow waters) & 0.2 & $10^{6}$ and $1.73 \times 10^{7}$ \\
\hline 5.0 (Deep waters) & 0.2 & $10^{4}, 10^{5}$ and $10^{6}$ \\
\hline
\end{tabular}

\subsection{Mesh and time-step dependence tests}

Mesh and time-step dependence tests are carried out for shallow and deep waters respectively at the highest Grashof number of each set $\left(G r=1.73 \times 10^{7}\right.$ for shallow waters and $G r=10^{6}$ for deep waters). Three meshes of $121 \times 61,201 \times 61$ and $301 \times 91$ are tested. It is found that all three meshes produce similar results in terms of the overall response of the flow to the periodic forcing. The results presented in the following sections are obtained using the medium mesh $(201 \times 61)$ and a dimensionless time step of $10^{-6}$.

\section{Flow Response to Periodic Forcing}

In this section, the diurnal flow in the model reservoir in response to the periodic thermal forcing is described for shallow and deep waters respectively.

\subsection{Flow response in shallow waters $(\boldsymbol{\eta}=\mathbf{0 . 6 2})$}

The numerical results obtained for $G r=1.73 \times 10^{7}$ are reported here. The simulation covers 10 full thermal forcing cycles in order to minimize the effect of the start-up flow, and the results of the last cycle are presented.

3.1.1 Flow response to daytime heating The daytime heating process corresponds to the first-half of each forcing cycle. Figure 3 plots the temperature contours and the corresponding streamlines at representative time instances. The times indicated in this figure are relative to the end of the ninth or the beginning of the tenth forcing cycle. In the streamline plots, the solid lines indicate a counter-clockwise circulation. Conversely, dashed lines indicate a clockwise circulation. 

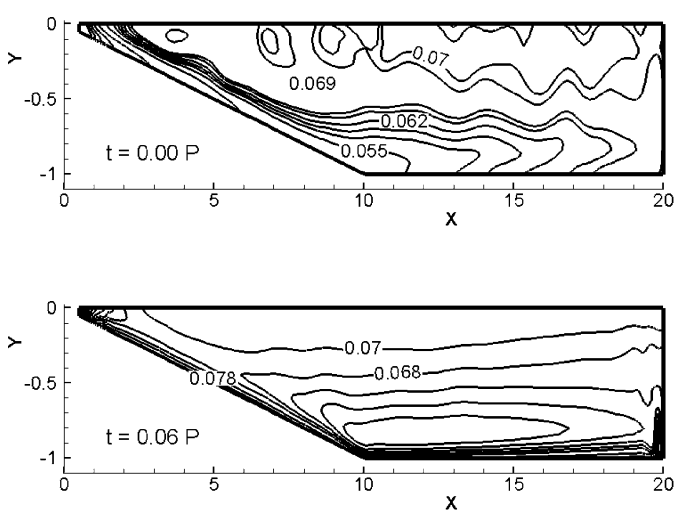

(b)

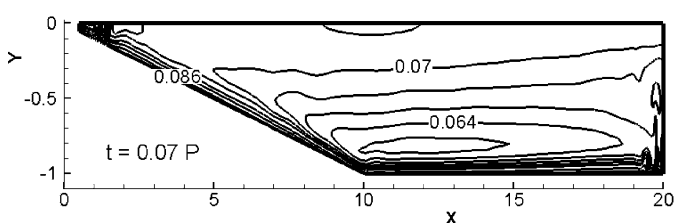

(c)

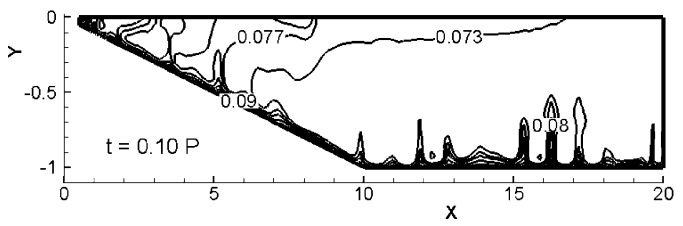

(d)

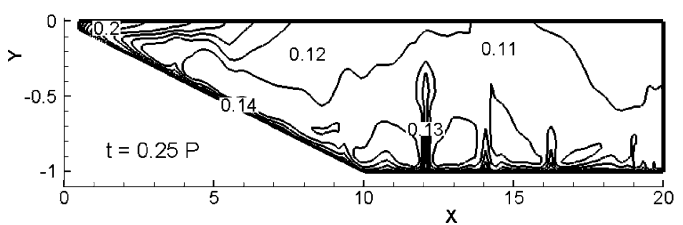

(e)

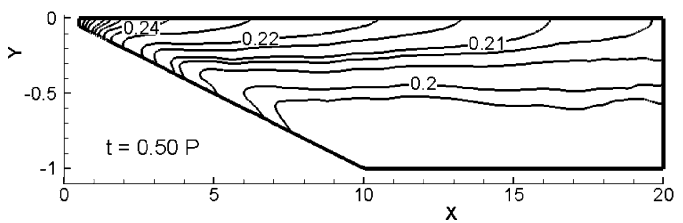

$(f)$
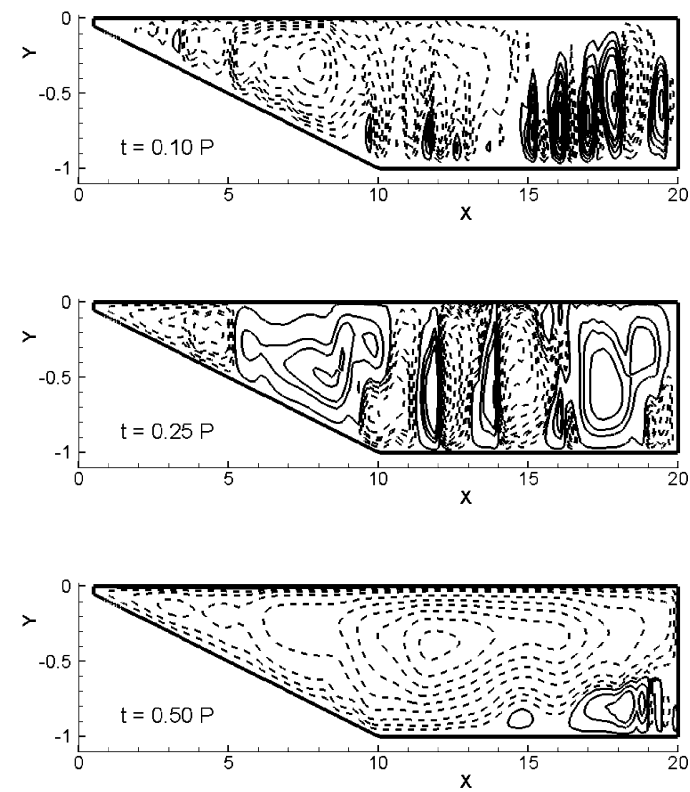

Fig. 3 Flow response to radiative heating at different stages in shallow waters $(\eta=0.62$, $\left.G r=1.73 \times 10^{7}\right)$. Left: temperature contours. Right: streamlines

At the beginning of this cycle $(t=0.00 P)$, the surface cooling is switched off and the radiation heating is switched on at the same time. However, residual temperature and flow structures formed in the previous cooling phase are still present. The temperature contours in Fig. 3 (a) clearly show that a cold gravity current is flowing downward along the sloping bottom and into the main water body along the flat bottom. The downward gravity current is a major feature expected in coastal waters during the night-time cooling process. Corresponding to this downward flow, a cavity-wide large-scale circulation is present, as can be seen from the streamlines in Fig. 3 (a).
It is also seen in Fig. 3 (a) that the cavity-wide circulation has multiple cores, which are the residual effect of plunging plumes formed during the preceding cooling phase.

As the radiative heating intensifies, the water temperature across the entire domain increases as a consequence of the absorption of the radiation entering from the water surface. Of particular interest is the growth of a thermal boundary layer along the sloping and flat bottom (see the temperature contours in Fig. 3(b)). The thermal boundary layer forms because, in shallow waters, a significant portion of the radiation reaches the bottom (the so-called residual radiation) and is absorbed by the bottom. The 
absorbed energy is then re-emitted back into the water as a boundary heat flux. The combined effects of the bottom boundary heat flux and the topography in the sloping region result in an upward flow along the slope ${ }^{(8)}$, as indicated by the dashed streamlines in Fig. 3 (b). This upward flow is in the opposing direction to the residual flow formed during the previous cooling phase. It is seen in Fig. 3 (b) that, at this time, the reverse flow appears only in the region near the tip and the slope. In the region with a flat bottom, the bottom boundary heat flux does not produce any flow at this stage due to the absence of the topographical effect.

Further to the previous description, the thermal boundary layer continues to grow, and the region with the reverse flow expands toward the core (Fig. 3 (c)). At this time $(t=0.07 P)$, the flow in the region above the slope is almost reversed completely, and the residual flow has been pushed toward the deep-end wall. It is worth noting that the thermal boundary layer is stable until this time.

As the thermal boundary layer grows further, it eventually becomes unstable to Rayleigh-Benard type instabilities. As a consequence, rising hot-water plumes occur all the way along the bottom thermal boundary layer, as can be seen from the temperature contours in Fig. 3(d). The thermal instability in the form of rising plumes is the same as the instability observed in shallow waters under constant radiative heating ${ }^{(7),(9),(12),(13)}$. As the hot-water plumes move upward, they generate secondary flows by pushing and entraining the nearby fluid. The rising thermals also break up the residual circulation and form a cellular flow structure in the domain (see Fig. 3(d)).

The subsequent flow response in the domain is dominated by the occurrence of the thermal instability. Figure 3 (e) plots the temperature and flow structures at $t=$ $0.25 P$ when the intensity of the radiative heating peaks. The occurrence of the rising thermals is clearly seen in this figure, and accordingly, the cellular flow structure persists (see Fig. 3(e)). Another effect of the thermal instability in the form of rising thermals is to accelerate the vertical mixing in the domain, which in turn reduces the temperature gradient over the local water depth and tends to weaken the thermal instability.

After the time $t=0.25 P$, the intensity of the radiative heating starts to decline. However, it is observed that the bottom boundary heating mechanism discussed above, along with the associated thermal layer instability and the resulting cellular flow structure, persists for a relatively long time. Eventually, the thermal instability dies out when the radiation entering from the water surface becomes very weak. At this stage, the flow response is dominated by the topographic effect in the sloping region. Since the radiation entering from the surface is uniform across the length of the domain, but the water depth increases gradually in the sloping region, the averaged absorption of the radiation over the local water depth increases toward the tip. Therefore, a horizontal temperature gradient is established in the domain, which drives a cavity-wide circulation up the slope and out of the tip region along the surface. The representative temperature and flow structures at this stage is shown in Fig. 3 (f), which plots the contours of temperature and streamlines at $t=0.50 P$. Clearly, a temperature gradient exists along the slope and the water surface, and the water is stably stratified in the majority of the core region (refer to the isotherms in Fig. 3 (f)). The corresponding streamlines indicate a clockwise circulation across the entire domain.

The temperature and flow structures shown in Fig. 3 (f) also represent the residual structures from the daytime heating phase since the radiative heating is switched off and at the same time the surface cooling is switched on at $t=0.50 P$.

3.1.2 Flow response to night-time cooling The flow response during the second-half of the forcing cycle $(0.50 P<t<P)$ may be described in a similar way. For brevity, the detailed description is not included here. In general, the flow response during the surface cooling phase is very similar to the radiative heating phase. The major difference between the heating and cooling phases is that there is no thermal boundary layer growing along the bottom during the cooling phase. Instead, a thermal boundary layer grows underneath the water surface. It is this surface layer that eventually becomes unstable, and the thermal instability in the form of sinking cold-water plumes breaks the residual circulation and promotes vertical mixing. At the end of the cooling phase, temperature and flow structures similar to those shown in Fig. 3 (a) are formed, and the previously described flow response is repeated in the following cycle.

\subsection{Flow response in deep waters $(\eta=5)$}

With all the three Grashof numbers investigated for deep waters, the observed flow responses to the periodic thermal forcing are very similar. In the following presentation, only the numerical results obtained for $\mathrm{Gr}=10^{4}$ are reported and described. Similar to the case for shallow waters, the simulation covers 10 full thermal forcing cycles in order to minimize the effect of the start-up flow, and the results of the last cycle are presented. Figure 4 plots the temperature contours and the corresponding streamlines at representative time instances. The times indicated in this figure are relative to the beginning of the forcing cycle. In general, the flow response in deep waters exhibits many features that are different from those observed in shallow waters. The details of the flow response in deep waters are described below.

At the beginning of the cycle $(t=0.00 P)$, the surface cooling is switched off and the radiation heating is switched on at the same time. However, similar to the shallow waters case, residual temperature and flow struc- 


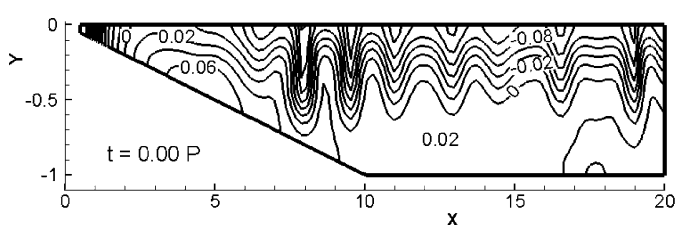

(a)

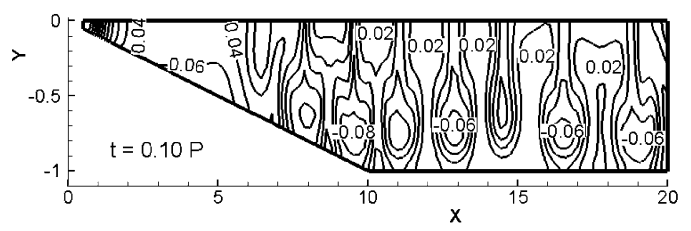

(b)

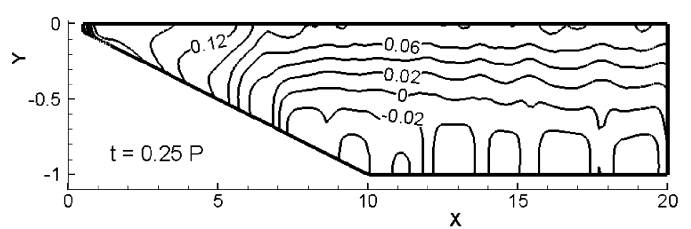

(c)

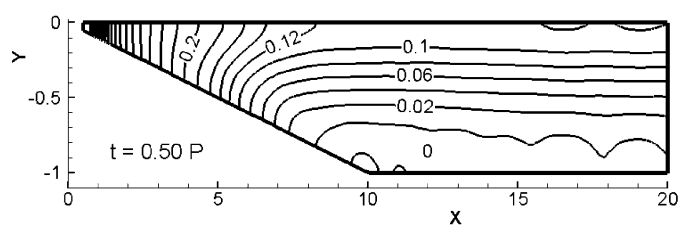

(d)
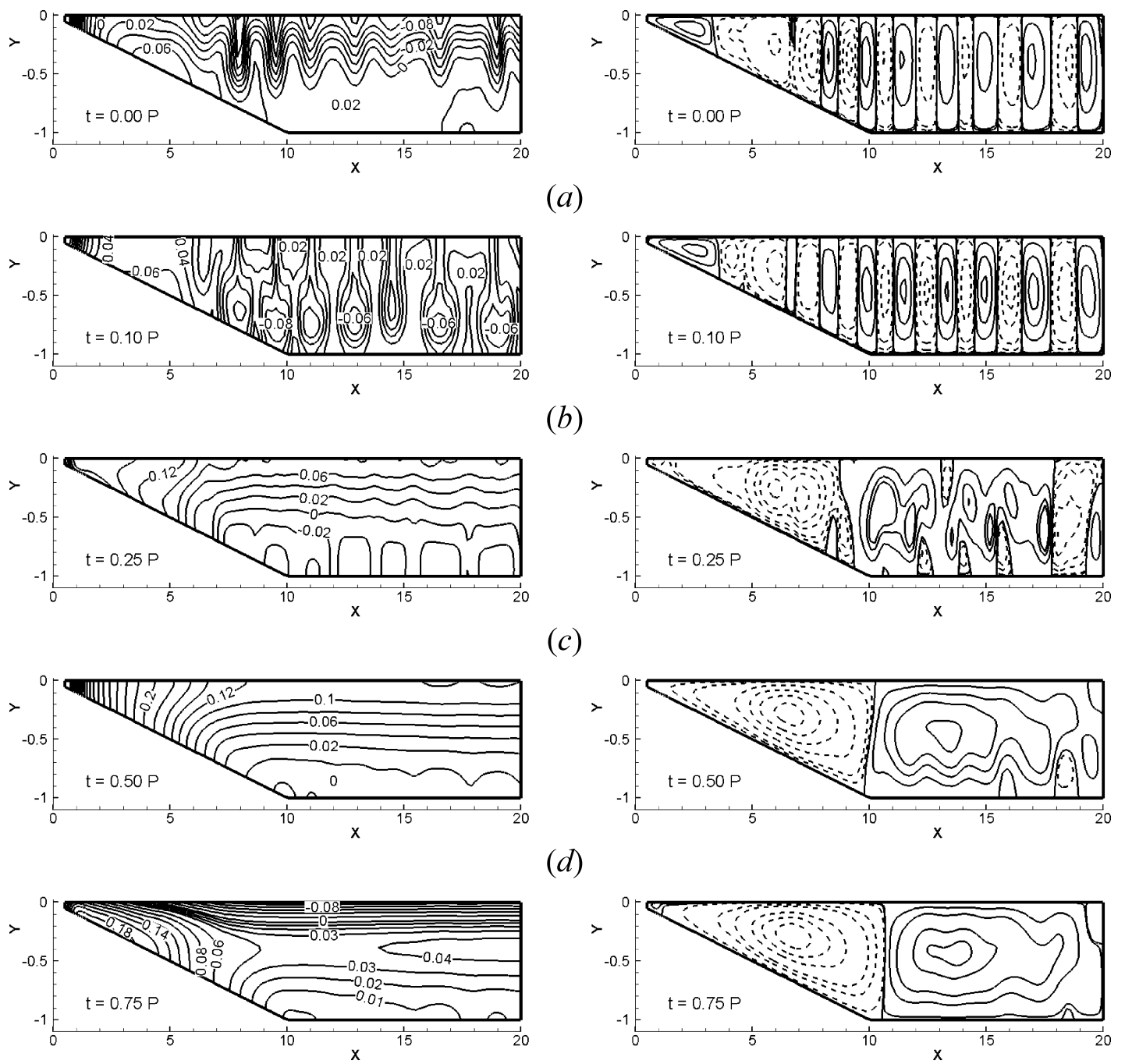

(e)
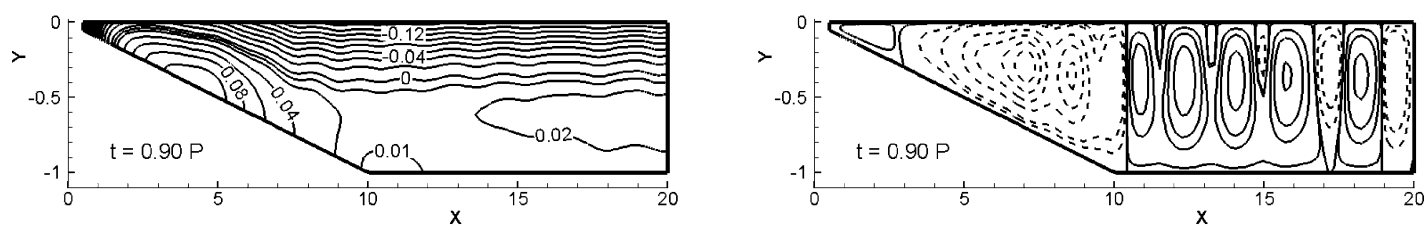

$(f)$
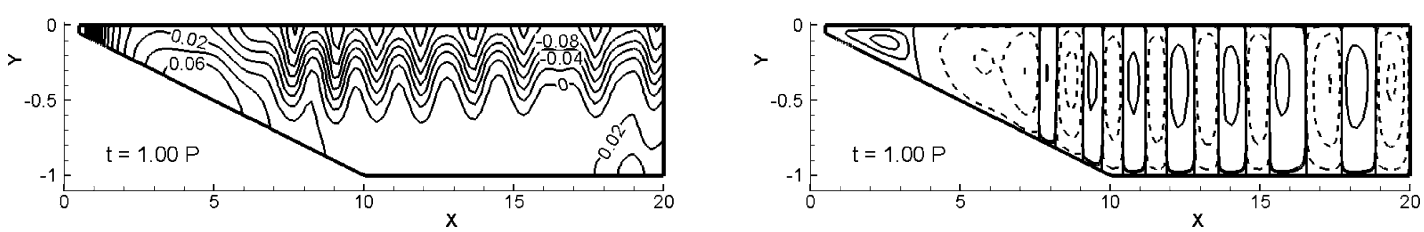

$(g)$

Fig. 4 Flow response to periodic heating and cooling at different stages in deep waters $(\eta=5$, $\left.G r=1.0 \times 10^{4}\right)$. Left: temperature contours. Right: streamlines

tures formed in the previous cooling phase are evident. It is seen in Fig. 4 (a) that the temperature structure in the reservoir consists of three distinct regions. In the region near the tip, the conduction effect is dominant, indicted by the approximately vertical isotherms. In the deep region, which covers the entire section with a uniform water depth and part of the section above the slope, plunging cold-water plumes are still forming. In the region between the tip and deep regions, a transitional region is present with stratification, which is potentially unstable but remains relatively stable at this time.

Corresponding to the above-described temperature 
structures, the streamline plot in Fig. 4 (a) also indicates three distinctive regions. In the tip region, an anticlockwise circulation is present with water flowing into the tip along the water surface and downward along the slope. In the transitional region, a clockwise circulation is evident. Therefore, an upwelling flow is formed between the tip and transitional regions, which is similar to that reported in Ref. (2). It will become clear later that the upwelling flow originates from the tip during the cooling phase, and moves gradually toward the deep region. In the deep region, a cellular flow pattern is formed due to the formation of plunging thermals, indicating the strong effect of vertical convection. A close examination of the flow field indicates that the circulations in the tip and transitional regions are very weak compared with that in the deep region.

The three-region temperature and flow structures remain unchanged until some time into the heating phase (see Fig. 4(b)). However, it is noticeable that the fluid layer under the water surface becomes warmer as a consequence of the absorption of the solar radiation. It is also noticed that the upwelling flow continues to move toward the deep region. This flow reaches its farthest position from the tip at about $t=0.10 P$.

As the radiative heating intensifies further, the upper water layer becomes stably stratified (Fig. 4(c)), and this stable layer gradually extended downwards (Fig. 4(d)). In the tip region, the vertical isotherms formed during the cooling phase are destroyed by the radiative heating (Fig. 4 (c)), and are eventually replaced by a similar temperature structure but with a converse horizontal temperature gradient (Fig. 4(d)). Associated with the development of the temperature structure is the evolution of the upwelling flow. It moves quickly back toward the tip along with the anticlockwise circulation after $t=0.10 P$. By the time the radiative heating reaches its peak intensity $(t=0.25 P)$, the upwelling flow disappears completely (Fig. 4 (c)). At this time, the flow in the slope region becomes fully in phase with the thermal forcing. That is, the flow moves up along the slope and out of the tip along the water surface. It is also noticeable in Fig. 4 that the previously described cellular flow pattern in the deep region formed during the cooling event is being replaced by a large anticlockwise circulation cell (Fig. 4 (c) and (d)). Since the circulations in the slope and deep regions are in the opposite directions, a downwelling flow is formed near the turning point along the bottom. This is the dominant feature for the rest of the heating phase.

The radiative heating ceases at $t=0.50 P$. In the meantime, the surface cooling starts. Despite of the formation of a cold layer under the water surface (refer to the isotherms in Fig. 4 (e)), the major flow structures formed during the previous heating event remain unchanged (see the streamlines in Fig. 4(e)). That is, the flow structure consists of two major circulating cells, a clockwise circu- lation above the slope, and an anticlockwise circulation in the deep region. The downwelling flow near the turning point is also distinct. At about $t=0.66 P$, an upwelling flow described previously starts to develop near the tip. As the surface cooling intensifies, the upwelling flow gradually moves out of the tip toward the deep region. This structure becomes evident at $t=0.75 P$ when the surface cooling reaches its peak intensity (Fig. 4 (e)).

As the surface cooling continues, the cold-water layer under the surface expands downwards and eventually becomes unstable. The instability is evidenced by the wavy patterns of the isotherms in Fig. 4 (f) and (g), which are the result of the formation of sinking cold-water plumes. As a consequence, a cellular flow structure is forming in the deep region (see the streamlines in Fig. 4 (f) and (g)). In the meantime, the temperature structure in the tip region formed during the previous heating phase is destroyed by the cooling event, and replaced by a similar structure with approximately vertical isotherms but a converse horizontal temperature gradient (refer to the isotherms in Fig. 4 (f) and $(\mathrm{g})$ ).

Associated with the above-described change of the temperature structure is the expansion of the anticlockwise circulation cell toward the deep region and the transport of the upwelling flow out of the tip (Fig. 4 (f) and (g)). The formation of plunging thermals and the transport of the upwelling flow toward the deep region are the major features for the rest of the cooling phase. By the time the surface cooling is switched off $(t=1.00 P)$, similar temperature and flow structures to those at the beginning of the forcing cycle are formed (Fig. 4(a) and (g)), and the flow development is continued in the following cycle.

\section{Horizontal Exchange Flow Rate}

The horizontal volumetric flow rate at a specified horizontal location is calculated in a 2D domain as ${ }^{(6),(8)}$

$$
Q(x)=\frac{1}{2} \int_{-h_{x}}^{0}|u| d y
$$

where $h_{x}$ is the local water depth at a given $x$ location, and $Q(x)$ quantifies the intensity of the horizontal exchange flow at the current location. Here, the dimensional exchange flow rate has been normalized using the scale $\sim \alpha$. By integrating $Q(x)$ along the horizontal direction as follows:

$$
Q=\frac{1}{L} \int_{0}^{L} Q(x) d x
$$

an averaged volumetric flow rate $Q$ is obtained. Here, $L$ is the total length of the domain.

Additional features of the flow response to the diurnal forcing can be observed from the time history of the calculated horizontal exchange flow rate, which is discussed below. 


\subsection{Calculated exchange flow in shallow waters $(\eta=0.62)$}

Figure 5 plots the time histories of the horizontal exchange flow rate calculated for two different Grashof numbers considered for shallow waters along with the corresponding thermal forcing. A positive value of the thermal forcing in Fig. 5 (a) represents the daytime radiative heating, whereas a negative value represents the night-time surface cooling. In Fig. 5, only the data obtained for the last five cycles (Cycles 6 to 10) are plotted. At $t / P=5$, the thermal forcing is switching from the night-time cooling to the daytime heating, and the intensity of the radiative heating increases for the following quarter of the cycle.

Consider first the case with $G r=1.73 \times 10^{7}$ shown in Fig. 5 (b). It is seen in this figure that, in contrast to the increasing thermal forcing, the averaged horizontal flow rate decreases from $t / P=5$ until $t / P=5.06$. This is due to the effect of the residual circulation formed in the precedent cooling phase. After $t / P=5.06$, the averaged horizontal flow rate starts to increase, which is in phase with the thermal forcing. The above observation indicates that the lag time of the flow response to the switch of the thermal forcing from cooling to heating is approximately $6 \%$ of the forcing period. As the radiative heating intensifies, thermal instabilities associated with the bottom heating start to occur, which cause the fluctuations of the horizontal exchange flow rate. It is seen in Fig. 5 (b) that the thermal instabilities dominate most of the heating phase and persist until well after the radiative heating peaks. When the radiative heating becomes sufficiently weak, the thermal instability dies out, and the overall flow rate decreases un-

(a)
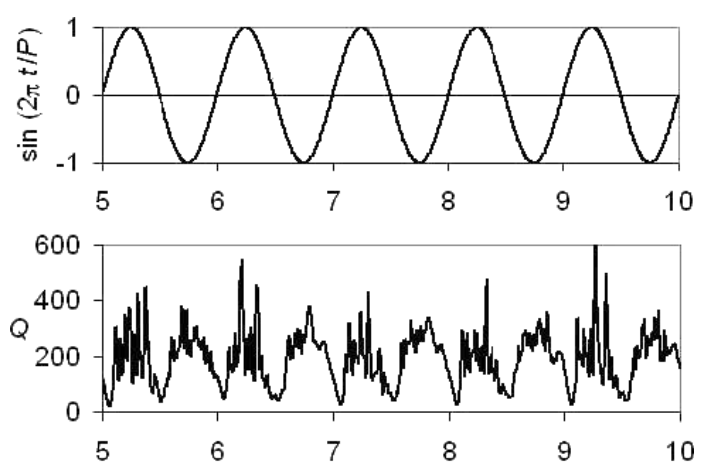

(c)

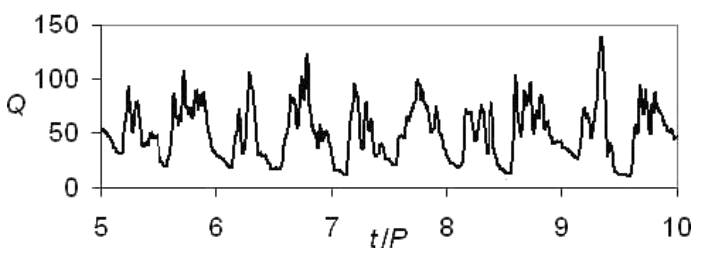

Fig. 5 Shallow water $(\eta=0.62)$ response to periodic thermal forcing. Plotted are the time histories of (a) the thermal forcing; (b) the averaged horizontal exchange flow rate for $G r=1.73 \times 10^{7}$; (c) the averaged horizontal exchange flow rate for $G r=10^{6}$ til a certain time after the cooling phase starts. Similarly, there is a lag of the reversal of the overall flow relative to the switch of the thermal forcing from heating to cooling, and the thermal instabilities dominate the flow response for most of the cooling phase.

It is also noticeable in Fig. 5 (b) that the overall strength of the circulation in the heating phase is approximately of the same order as that in the cooling phase. This is a special feature of the shallow water case. As will be seen in the following section, in deep waters, the circulation in response to the radiative heating is significantly weaker than the circulation in response to the surface cooling.

The time history of the averaged horizontal exchange flow rate obtained for $G r=10^{6}$ (see Fig. 5 (c)) exhibits similar features. However, three additional features in comparison with the higher Grashof number case are worth noting (see Fig. 5 (c)). First, the strength of the overall circulation is much weaker; second, the thermal instability becomes weaker, and the frequency is also reduced; finally, the lag time of the flow response to the switch of the thermal forcing is longer for the lower Grashof number case. The lag time for the switch from cooling to heating is estimated to be about $17 \%$ of the forcing period for $G r=10^{6}$.

\subsection{Calculated exchange flow in deep waters $(\eta=5)$}

The time histories of the calculated horizontal exchange flow rate in deep waters are plotted for different Grashof numbers in Fig. 6. Similar to the case of shallow waters, only the data obtained for the last five cycles (Cycles 6 to 10) are presented.

In general, the time histories of the calculated horizontal exchange flow rate for different Grashof numbers exhibit certain common features. First of all, the exchange flow rate shows a periodic behaviour in response to the periodic forcing. Secondly, the flow response is clearly out

(a)

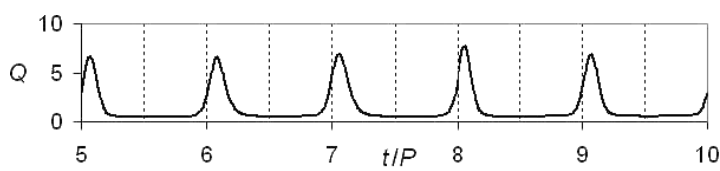

(b)

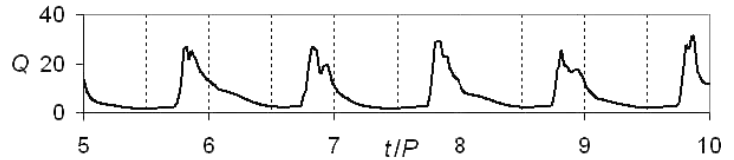

(c)

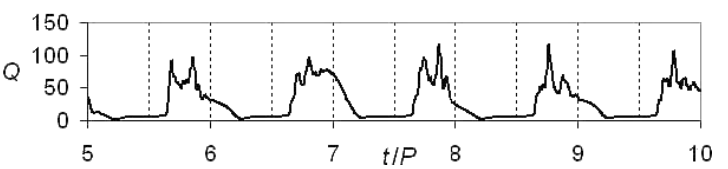

Fig. 6 Deep water $(\eta=5)$ response to periodic thermal forcing represented by the time histories of the calculated horizontal exchange flow rate for different Grashof numbers. (a) $G r=10^{4}$; (b) $G r=10^{5}$; (c) $G r=10^{6}$ 
of phase with the thermal forcing. Thirdly, within each cycle of the flow response, there is a time period with very weak flow response, and a period with very intensive flow response. The weak-response period corresponds to the delayed flow response to the radiative heating by solar radiation, and the intensive-response period corresponds to the delayed flow response to the cooling due to heat loss from the water surface. The flow response to the radiative heating is very weak because, in deep waters, most of the solar radiation entering from the water surface is absorbed within a relatively thin layer immediately below the water surface, which results in a stable stratification. Finally, the instability effect associated with the surface cooling is clearly shown. This effect is smoothed out for the lowest Grashof number case when the averaged horizontal exchange flow rate is calculated.

The dependence of the flow response in the reservoir on the Grashof number is also clearly demonstrated in Fig. 6. It is seen in this figure that the intensity of the flow response increases with the Grashof number. The same is true for the effect of the flow instability. Another noticeable feature in Fig. 6 is the shift of the peak flow response to the thermal forcing. For the lowest Grashof number case $\left(G r=10^{4}\right)$, the peak appears during the radiative heating phase, whereas for the higher Grashof number cases $\left(G r=10^{5}, 10^{6}\right)$, the peak appears during the cooling phase. The shift of the peak response is coincident with the variation of the time lag of the flow response to the thermal forcing. From the previous description of the flow response for $G r=10^{4}$, it is estimated that it takes approximately a quarter of the cycle for the flow above the slope to completely reverse after the thermal forcing is switched from cooling to heating. There is no complete reversal of the flow above the slope after the switch from heating to cooling. However, based on the appearance of the peak flow response in Fig. 6, the time lag of the flow response relative to the switch from the radiative heating to the surface cooling is estimated to be about $0.35 P$ for $G r=10^{4}$. As the Grashof number increases, the time lag of the flow response to the switches of the thermal forcing is reduced.

\section{Summary}

The flow response to diurnal thermal forcing in reservoirs with slowly varying topography is described based on numerical simulations. The present numerical results have revealed major distinctive features of the response flow in shallow and deep reservoirs.

In the shallow reservoir, a significant amount of the solar radiation entering from the water surface penetrates all the way along the local water column and reaches the bottom. The residual radiation is then absorbed by the bottom. The absorption of the residual radiation and the release of the absorbed energy by the bottom provide a dominant bottom heating mechanism that drives a flow in response to the thermal forcing. The response flow to the daytime radiative heating is comparable with the response flow to the night-time cooling.

In the deep reservoir, the solar radiation entering from the surface is mostly absorbed by a relatively thin layer under the surface, resulting in a stable stratification in the majority of the water body. The bottom heating mechanism discussed above is only present in a small region near the tip, where the local water depth is less than the penetration depth of the solar radiation. Therefore, the response flow to the daytime radiative heating is very weak compared with that in the shallow reservoir. The flow response to the switch from the surface cooling to the radiative heating in the deep reservoir is characterized by the reversal of the flow above the slope and stable stratification in the deep region, and the flow response to the switch from the radiative heating to the surface cooling is characterized by the appearance of an upwelling flow near the tip and the formation of plunging thermals in the deep region. Figures 5 (c) and 6(c) indicate that the response exchange flow to the night-time surface cooling is of the same order in the shallow and deep reservoirs for the same Grashof number.

The present numerical simulations have also revealed a clear time lag of the flow response to the switch of the thermal forcing between the daytime radiative heating and the night-time surface cooling. In the shallow reservoir case, the estimated time lag is significantly less than a quarter of the cycle, whereas in the deep reservoir the estimated time lag is beyond a quarter of the cycle and depends largely on the Grashof number. The deep water result is in a good qualitative agreement with that reported in Ref. (2).

It is also observed in this study that the thermal instabilities associated with the bottom heating during the daytime radiative heating phase and those associated with the surface cooling during the night-time cooling phase play an important role in breaking the residual circulation and reversing the flow in the shallow reservoir. In the deep reservoir, the thermal instabilities associated with bottom heating are not prominent. However, the instabilities in the form of plunging thermals during the night-time cooling phase are still prominent, and these instabilities are also the dominant mechanism responsible for vertical mixing in field situations.

The response of the flow to the changing forcing demonstrated here may have important implications for the distribution of suspended materials or the response of suspended biological materials in the water. In particular, the behaviour of algal populations is sensitive to both the light climate and the local populations of nutrients and to the local temperature. The complex flow patterns and the lag in response to the thermal forcing may strongly influence the photosynthetic response of algae, and thus may 
be a factor in the eutrophic state of a water body.

\section{Acknowledgement}

This research was supported by the Australian Research Council (ARC), the Australian Partnership for Advanced Computing (APAC) and Queensland Parallel Supercomputing Foundation (QPSF).

\section{References}

( 1 ) Sturman, J.J., Oldham, C.E. and Ivey, G.N., Steady Convective Exchange Flows Down Slopes, Aquatic Sciences, Vol.61 (1999), pp.260-278.

( 2 ) Farrow, D.E. and Patterson, J.C., On the Response of a Reservoir Sidearm to Diurnal Heating and Cooling, Journal of Fluid Mechanics, Vol.246 (1993), pp.143161.

( 3 ) Farrow, D.E., Periodically Forced Natural Convection over Slowly Varying Topography, Journal of Fluid Mechanics, Vol.508 (2004), pp.1-21.

( 4 ) Horsch, G.M. and Stefan, H.G., Convective Circulation in Littoral Water due to Surface Cooling, Limnology \& Oceanography, Vol.33 (1988), pp.1068-1083.

( 5 ) Horsch, G.M., Stefan, H.G. and Gavali, S., Numerical Simulation of Cooling-Induced Convective Currents on a Littoral Slope, International Journal for Numerical Methods in Fluids, Vol.19 (1994), pp.105-134.

( 6 ) Lei, C. and Patterson, J.C., Unsteady Natural Convection in a Triangular Enclosure Induced by Surface Cooling, International Journal of Heat and Fluid Flow,
Vol.26 (2005), pp.307-321.

( 7 ) Lei, C. and Patterson, J.C., Natural Convection in a Reservoir Sidearm Subject to Solar Radiation: A Two-Dimensional Simulation, Numerical Heat Transfer, Part A: Applications, Vol.42, No.1 (2002), pp.1332.

( 8 ) Lei, C. and Patterson, J.C., Unsteady Natural Convection in a Triangular Enclosure Induced by Absorption of Radiation, Journal of Fluid Mechanics, Vol.460 (2002), pp.181-209.

(9) Lei, C. and Patterson, J.C., A Direct ThreeDimensional Simulation of Radiation-Induced Natural Convection in a Shallow Wedge, International Journal of Heat and Mass Transfer, Vol.46 (2003), pp.11831197.

(10) Adams, E.E. and Wells, S.A., Field Measurements on Side Arms of Lake Anna, Va., Journal of Hydraulic Engineering, Vol.110 (1984), pp.773-793.

(11) Monismith, S.G., Imberger, J. and Morison, M.L., Convective Motions in the Sidearm of a Small Reservoir, Limnology \& Oceanography, Vol.35 (1990), pp.16761702.

(12) Lei, C. and Patterson, J.C., Natural Convection in a Reservoir Sidearm Subject to Solar Radiation: Experimental Observations, Experiments in Fluids, Vol.32, No.5 (2002), pp.590-599.

(13) Lei, C. and Patterson, J.C., A Direct Stability Analysis of a Radiation-Induced Natural Convection Boundary Layer in a Shallow Wedge, Journal of Fluid Mechanics, Vol.480 (2003), pp.161-184. 